\title{
ROUTING WITH NEW NODE BALANCING PROTOCOL
}

\author{
G.Raghu Ram ${ }^{1}$, S.Anuradha ${ }^{2}$ and A.Subramanyam ${ }^{3}$
}

\begin{abstract}
Implementation of new node balancing protocol named MDBR algorithm to control the congestion, along with its design and its application on different network topologies with varied load of data packets is discussed in present paper.

Keywords - Node balancing, stigmergy, Ant colony optimization, Networks.
\end{abstract}

\section{INTRODUCTION}

Congestion control over network, for all types of media traffic, has been an active area of research in the last decade [1]. This is due to the flourishing increase in the audiovisual traffic of digital convergence. There exists a variety of network applications built on its capability of streaming media either in real-time or on demand such as video streaming and conferencing, voice over IP (VoIP), and video on demand (VoD). The number of users for these network applications is continuously growing hence resulting in congestion.

All the networks applications do not use TCP and therefore do not allow fair allocation with the available bandwidth. Thus, the result of the unfairness of the non-TCP applications did not have much impact because most of the traffic in the network uses TCP-based protocols. However, the quantity of audio/video streaming applications such as Internet audio and video players, video conferencing and analogous types of real-time applications is frequently increasing and it is soon expected that there will be an increase in the proportion of non-TCP traffic. In view of the fact that these applications commonly do not amalgamate TCPcompatible congestion control mechanisms, network applications treat challenging TCPflows in an unreasonable manner. All TCP-flows reduce their data rates in an attempt to break up the congestion, where the non-TCP flows maintains to send at their original rate. This highly unfair condition will lead to starvation of TCP-traffic i.e.., congestion collapse [2], [3], which describes the disagreeable situation where the accessible bandwidth in a network is almost entirely occupied by packets which are discarded because of the congestion before they reach their destination. For this reason, it is desirable to define suitable congestion control mechanisms for non-TCP traffic that are compatible with the rateadaptation mechanism of TCP. These mechanisms should make non-TCP applications TCPfriendly, and thus lead to a fair distribution of bandwidth. Unicast is a one-to-one form of communication in networks where multicast is one-to-many. Multicast is advantageous over Unicast particularly in bandwidth reduction, but Unicast is until the extensively widen communication form network. congestion and optimize the routing technique with a new searching method named MDB routing.

\section{GOALS AND METRICS OF CONGESTION CONTROL}

\footnotetext{
${ }_{1}^{1}$ Dept. of Computer Science, Rayalaseema University Kurnool, A.P, India.

${ }^{2}$ Vardhaman College of Engg. Kacharam, Shamshabad, Hyderabad, Telengana

${ }^{3}$ AITS, Rajampet, YSR dt. Andhra Pradesh, India.
} 


\section{A) Goals that are taken for the evaluation process of a congestion control algorithm is:}

i. To congregate to fairness quickly and efficiently. ii. To sustain a high responsiveness.

iii. To coexist fairly and be compatible with long established widely used protocols.

\section{B ) APPLYING THE DB SEARCH FOR ANT ALGORITHMS}

Ants are capable of navigating complex terrains in search of food. They also find their way back to the nest. Over a period of time a colony of ants are able to find the shortest path between the food source and the nest by laying pheromones (a chemical substance) and give way over a period of time. All ants choose to move over tracks of high pheromone concentration along it. In the beginning each ant goes in search of food and as they move the pheromone is laid along the path. When an ant finds the food source it starts its return journey along the same path and adds to the pheromone concentration along it. Since the colony comprises of a large number of ants, a parallel search ensues. Chances are that several of them discover the food different paths. Naturally the ant that found the closest path (out of all those discovered so far) would over a period of time shuttle up and down more number of times than its counterparts. This period of time the shortest path exists while other paths fade away due to pheromone volatility. In the real world ants update their pheromone trails on the fly. In the world of optimization, this method may be tweaked or changed to suit the search domain. Trails could be updated after the ant discovers a solution path. Such trails could be updated based on the fitness of the solution found. The pheromone laid along the paths could be proportional to its fitness. While searching the food source ants doesn't follow any specific searching technique (DFS and BFS). All the ants spreads through out the network in search of food source some will be scattered using DFS and some will be moving by BFS and some will be moving towards food by both the ways(DFS and BFS) to reach the food source in shorter period. This will be proved by the ACO algorithms for TSP (Traveling sales man problem). The construction graph $\mathrm{G}=(\mathrm{C}, \mathrm{L})$, where the set $\mathrm{L}$ fully connects the components $\mathrm{C}$, is identical to the problem graph, that is $\mathrm{C}=\mathrm{N}$ and $\mathrm{L}=\mathrm{A}$. the set of states of the problem corresponds to the set of all possible partial tours, and the constraints ' $\Omega$ enforce that the ants construct only feasible tours that correspond to permutations of the city indices. This is always possible, because the construction graph is complete graph and any closed path that visits all the nodes without repeating any node corresponds to feasible tour. The pheromone trails are associated with the arcs and therefore $\square \mathrm{ij}$ refers to the desirability of visiting city $\mathrm{j}$ directly after city $\mathrm{i}$. the heuristic information is chosen as $\eta \mathrm{ij}=1 / \mathrm{d} \mathrm{ij}$, the heuristic desirability of going from city $\mathrm{i}$ directly to city $\mathrm{j}$ is inversely proportional to the distance between the two cities.

Tours are constructed by applying the simple constructive procedure to each ant.

1. Choose according to some criterion, a start city at which the ant is positioned.

2. Use pheromone and heuristic values to probabilistically construct a tour by iteratively adding cities that the ant has not visited yet until all cities have been visited.

3. Go back to the initial city. After all ants have completed their tour, they may deposit pheromone on the tours they have followed. 


\section{A. Defining the path construction in DB Routing Algorithm}

Identify applicable sponsor/s here. If no sponsors, delete this text box. (sponsors) In Ant colony System, m artificial ants concurrently build a tour of the TSP. Initially, ants are put on randomly chosen cities. At each construction step, ant $\mathrm{k}$ applies a probabilistic action choice rule, called proportional rule, to decide which city to visit next. In particular, the probability with which ant $\mathrm{k}$, currently at city $\mathrm{I}$, chooses to go to city $\mathrm{j}$ is $\mathrm{P} \mathrm{k} i \mathrm{ij}=[\square \mathrm{ij}] \alpha$ [ $\eta \mathrm{ij}] \beta$ if j $\in \mathrm{N}$ i k

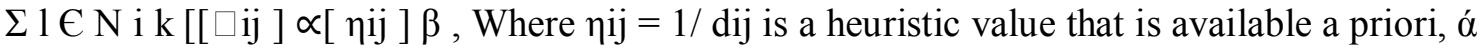
and $\beta$ are two parameters which determine the relative influence of the pheromone trail and the heuristic information and $\mathrm{N} \mathrm{i} \mathrm{k}$ is the feasible neighborhood of ant $\mathrm{k}$ when being at city $\mathrm{I}$, the set of cities that ant $\mathrm{k}$ has not visited yet. By this probabilistic rule, the probability of choosing a particular $\operatorname{arc}(\mathrm{I}, \mathrm{j})$ increases with the value of the associated pheromone trail $\square \mathrm{ij}$ and of the heuristic information value $\eta \mathrm{ij}$. If $\alpha=0$, the closest cities are more likely to be selected . if $\beta=0$, only pheromone amplification is at work I,e only pheromone is used, with out any heuristic bais. This generally leads to rather poor results and in particular, for values of $\alpha>1$ it leads to the rapid emergence of the stagnation i.e. all the ants follow the same path and construct the same tour. Good parameter values are as follows $\alpha=1, \beta=2$ to $5, \mathrm{P}=0.5$, $\mathrm{m}=\mathrm{n}, \square 0=\mathrm{m} / \mathrm{cmn}$. Here, $\mathrm{n}$ is the number of cities in a TSP instance.

\section{MODIFICATIONS PROPOSED TO DB ROUTING ALGORITHM}

Original DB routing algorithm focuses only on the searching method used in finding out the shorter paths in the network but not on node balancing concept. Based on the original DB routing algorithm few modifications are suggested to be added to enhance its performance.

\section{A. Congessition Control (Control of the Number of Ants in the Network)}

For every algorithm, the network load generated by routing packets is stored as the ratio between the bandwidth occupied by all the routing packets and the total available network bandwidth. The routing overhead is the main function of the topological properties of the network and of the generation rate of the routing information packet. Ant Net produces a routing overhead depending on the ants' generation rate and the length of the path along they travel. As the followed path of routing ant grows (either because of topology or bad routing) the routing overhead grows. The DB routing and original Ant Net does not take into account the generated routing overhead and its effect on overall network performance.

\section{B. Introducing Load Balancing Technique to over come the above mentioned problem}

Original DB routing algorithm addresses the routing problems but not load balancing [11]. Load balancing is heavily relied on routing; Ant Net routing philosophy can lead to network congestion, high delay and may create deadlock. For example a node that lies on several routes will have a large number of packets for different destinations in its interface queue; all these packets will experience high queuing delays resulting in a high overall end to end delay. Load balancing technique is needed to remove such bottlenecks. The optimal solution found in the first phase is then finalized by a deterministic procedure that adjusts flows in order to achieve the precise balance of the input-output flow at each node. An outline of this procedure is shown as follows 
Modified DB algorithm for direct representation. For any node if its total input flow is bigger than its total output flow, it finds a path from the given node to the source node, and then decreases the flow along that path as much as possible. This might be repeated several times for each node, until the excess input flow has been completely removed.

input: Flows that already satisfy the condition that at each inner node the sum of its output flows is at most as big as the sum of its input flows.

output: Balanced flows for which the balance condition holds is that every inner node has its total output flow equal to its total input flow.

1 while (exists node $\mathrm{v}$ with unbalanced in-out flows)

2 flow difference $=\mathrm{v}$. in $-\mathrm{v}$. out

3 do

4.1 Find an acyclic path $\mathrm{P}$ from $\mathrm{v}$ to the source node $\mathrm{s}$ such that all edges in the path are assigned a positive flow.

4.2 Set minimize to the flow of the edge with the minimal flow along the path $\mathrm{P}$

4.3 minimize $=\min ($ minimize, flow diff $)$

5 Decrease flows of all edges of path $\mathrm{P}$ by value of minimize

6 flow diff $=$ flow diff - minimize

7 while (flow diff $>0$

\section{IV.EXPERIMENTS \& RESULTS}

Here 12 different experiments were executed on 4X4, 5X5 and 10X10 grid network topologies using MDB routing algorithm and DB algorithm as benchmark with different network load as 1000, 3500, 5000 and 10000 packets and compared the results. The comparative results were displayed in the table1.

Experiment I: Four simulations were done on $4 \times 4$ grid with $1000,3500,5000$ and 10000 as packet load for both the MDB and DB routing algorithms was executed.

Experiment II : Four simulations were done on 5x5 grid with 1000, 3500,5000 and 10000 as packet load for both the MDB and DB routing algorithms was executed.

Experiment III: Four simulations were done on 10x10 grid with 1000, 3500,5000 and 10000 as packet load for both the MDB and DB routing algorithms was executed.

Table 1. Comparative results of MDB and DB Routing algorithms

\begin{tabular}{|c|c|c|c|c|c|c|}
\hline & \multicolumn{3}{|c|}{ DB Routing Algorithm } & \multicolumn{3}{|c|}{ MDB Routing Algorithm } \\
\hline Topology & $4 \mathrm{X} 4$ & $5 \times 5$ & $10 \times 10$ & $4 \mathrm{X} 4$ & $5 \times 5$ & $10 \times 10$ \\
\hline No. Of Packets & 1000 & 1000 & 1000 & 1000 & 1000 & 1000 \\
\hline Dead & 494 & 667 & 890 & 491 & 658 & 556 \\
\hline $\begin{array}{l}\text { Average time for } \\
\text { packet }\end{array}$ & 235.94 & 251.68 & 268.41 & 206.5 & 249.43 & 256.9 \\
\hline No. Of Packets & 3500 & 3500 & 3500 & 3500 & 3500 & 3500 \\
\hline Dead & 694 & 548 & 268 & 494 & 348 & 160 \\
\hline $\begin{array}{l}\text { Average time for } \\
\text { packet }\end{array}$ & 316.13 & 343.80 & 422.22 & 306.53 & 333.98 & 412.71 \\
\hline No. Of Packets & 5000 & 5000 & 5000 & 5000 & 5000 & 5000 \\
\hline
\end{tabular}




\begin{tabular}{|l|l|l|l|l|l|l|}
\hline Dead & 3429 & 2388 & 898 & 2492 & 1382 & 790 \\
\hline $\begin{array}{l}\text { Average time for } \\
\text { Packet }\end{array}$ & 239.79 & 268.81 & 436.36 & 219.77 & 248.44 & 446.31 \\
\hline & \multicolumn{7}{|l|}{} \\
\hline No. Of Packets & $\mathbf{1 0 0 0 0}$ & $\mathbf{1 0 0 0 0}$ & $\mathbf{1 0 0 0 0}$ & $\mathbf{1 0 0 0 0}$ & $\mathbf{1 0 0 0 0}$ & $\mathbf{1 0 0 0 0}$ \\
\hline Dead for & 219.89 & 394.11 & 463.32 & 199.84 & 335.18 & 443.34 \\
\hline $\begin{array}{l}\text { Average time } \\
\text { Packet }\end{array}$ & & & & & & \\
\hline
\end{tabular}

\section{CONCLUSION}

A surprising observation is that MDB routing algorithm is able to route the packages faster through the network than the DB algorithm. The reason for this is the difference in the way the traffic is distributed. The MDB algorithm distributes the traffic by utilizing most of the routers . This also controls the packet death while transmitting the data packets. The performance of the routing algorithms is measured by two factors. First the average time taken for data transmission of packet and other depends on the death rate of the packet while transmitting. With the above results we conclude that MDB routing algorithms performs better than the DB routing algorithm with less average time of the packet and less dead packets (table 1) With the concept of load balancing in the DB as Modified DB (MDB) one can improve the speed of data transmission, can control the death rate and finally improving the throughput

\section{ACKNOWLEDGMENT}

The Part of this work is supported by Post Doctoral Fellow awardee Anuradha Sanike (No. F.15-1/2014-15/PDFWM-2014-15-GE-AND-26672) by University Grants Commission (UGC), New Delhi, India.

\section{REFERENCES}

[1] Marco Dorigo \& Thomas Stutzle - "Ant Colony Optimization" - MIT press edition, year: 2004.

[2] Routing - [Online Article] - Accessed on: 03.06.2011. - Link: http://en.wikipedia.org/wiki/Routing

[3] Dijkstra's algorithm - [Online Article] - Accessed on: 03.06.2011. Link:http://en.wikipedia.org/wiki/Dijkstra\%27s_algorithm Prim's algorithm - [Online article] - Accessed on: 02.06.2011. IEEE

[4] Mcgraw-Hill's Advanced Topics in Computer Science Series, New Ideas in Optimization.

[5] Gianni Di Caro - "Ant colony optimization and its application to Adaptive routing in telecommunication networks" - year: 2004.

[6] M. Dorigo, V. Maniezzo \& A. Colorni, 1996. "Ant System: Optimization by a Colony of Cooperating Agents", IEEE

[7] S.Anuradha, G.RaghuRam, K.E.Sreenivasa murthy, “ Evolution Of New Searching Technique In Unicast Routing Algorithm Of Data Networks" Praise Worthy Prize's International Review on Computers and Software(IRECOS) Voume.4,n`3, May 2009,pages(321-330).

[8] S.Anuradha, G.Raghu Ram, "Dynamic Congestion Control using MDB-Routing Algorithm “ Springer's Institute of Engineers India-B(IEI-B)series Journal May 2014.

[9] Gianni Di Caro, Marco Dorigo, AntNet: distributed stigmergetic control for communication networks, Journal of Artificial Intelligence Research 9 (1998) 317-365.

[10] M. Welzl, W. Eddy, Congestion Control in the RFC Series, University of Innsbruck, October 30, 2012

[11] S. Anuradha, "New Routing Technique to improve transmission Speed of Data Packets in Point to Point Networks" ICGST Inter National Journal on computer Networks and Internet Research(CNIR) Volume 8,Issue II January 2009,Pages(1..10) 
[12] Elke Michlmayr, Arno Pany, Sabine Graf: “Applying Ant-based Multi- Agent Systems to Query Routing in Distributed Environments," 3rd IEEE Conference On Intelligent Systems (IEEE IS06), London, UK, September 2006

[13] Elke Michlmayr: "Ant Algorithms for Search in Unstructured Peer-to- PeerNetworks," Ph.D.Workshop, 22nd International Conference on Data Engineering (ICDE 2006), Atlanta, Georgia, USA, April 2006

[14] S. Anuradha, , K.E. Sreenivasa murthy, B. Gurunath Reddy, "Fast Transfer of Packets Through DB Routing Using Ant Colony Optimization” Praise Worthy Prize's International Review on Computers and Software(IRECOS) Vol.3 N.4 July 2008, Pages(349..355)

[15] Lilia Rejeb and Zahia Guessoum. The Exploration-Exploitation Dilemma for Adaptive Agents. In Proceedings of the 5th European Workshop on Adaptive Agents and Multi-Agent Systems (AAMAS05), March 2005

[16] S.Anuradha, G.RaghuRam, K.E.Sreenivasa murthy, V.Raghunath Reddy, B.Satya narayana "Genesis of DB Routing Algorithm in Unicasting Networks" ICGST Inter National Journal on computer Networks and Internet Research(CNIR) Volume 9, Issue I, July 2009, Pages (47.. 55)

[17] St " utzle and Marco Dorigo. A short convergence proof for a class of ant colony optimization algorithms. IEEE Transactions on Evolutionary Computation, 6(4):358-365, August 2002

[18] S.Anuradha, G.RaghuRam, K.E.Sreenivasa murthy, V.Raghunath Reddy, B.Satya narayana “Evolution Of New Searching Technique In Unicast Routing Algorithm Of Data Networks" Praise Worthy Prize's International Review on Computers and Software(IRECOS) Voume.4,n³ ,May 2009,pages(321-330)

[19] Dorigo, Marco and Stützle, Thomas. (2004) Ant Colony Optimization, Cambridge, MA: The MIT Press

[20] W. Chung, -Congestion control for streaming media,|| Ph. D. dissertation, Polytechnic Inst., Worcester, 2015.

[21] S. Floyd, Ed., "Metrics for the Evaluation of Congestion Control Mechanisms", March 2008.

[22] De Marco, F. Postiglione, M. Longo, -Run-time adjusted congestion control for multimedia: experimental results\|, Journal of Interconnection Networks (JOIN), vol. 5, no. 3, pp. 249-266, 2004.

[23] A. Kuzmanovic, A. Mondal, S. Floyd and K. Ramakrishnan "Adding Explicit Congestion Notification (ECN) Capability to TCP's SYN/ACK Packets" AT\&T Labs Research, June 2014. 